\title{
Should we continue to use the 75-g OGTT to diagnose diabetes?
}

\author{
Maria Tomkins $^{1} \cdot$ Diarmuid Smith $^{1}$
}

Published online: 14 November 2019

(C) Royal Academy of Medicine in Ireland 2019

In 2010 the WHO/ADA allowed the use of the HbAlc to diagnose diabetes (Table 1) [1]. Clinicians had long called for the introduction of the $\mathrm{HbA} 1 \mathrm{c}$ as a diagnostic tool for diabetes as the $75 \mathrm{~g}$ OGTT had started to fall out of favour. The OGTT is a cumbersome test with several conditions required to complete the test satisfactorily; patients are required to be on a stable diet of greater than $150 \mathrm{~g}$ of carbohydrate per day for 3 days preceding the OGTT. Patients have to fast overnight, give a fasting blood sample, then ingest the oral $75 \mathrm{~g}$ glucose load over five minutes, sit still for $2 \mathrm{~h}$ frequently in an already busy GP surgery with a second blood sample taken at $2 \mathrm{~h} \mathrm{[2].} \mathrm{The} \mathrm{OGTT} \mathrm{is} \mathrm{altered} \mathrm{by} \mathrm{an} \mathrm{acute} \mathrm{episode} \mathrm{of}$ stress, illness, or exercise and has a high rate of intraindividual variability with a coefficient of variability $(\mathrm{CV})$ of up to $5.7 \%$ of the fasting plasma glucose and $16.7 \%$ on the $2-\mathrm{h}$ sample [3]. Therefore, if a patient with glucose intolerance undergoes testing with an OGTT they could be diagnosed with T2DM one day and impaired glucose tolerance (IGT) on another day. It is estimated given the variability in the OGTT that $>12 \%$ of patients who undergo OGTT are misclassified as either diabetes or IGT, a confusing situation for both patient and doctor [3].

1. Fasting plasma glucose $(\mathrm{FPG}) \geq 7.0 \mathrm{mmol} / \mathrm{L} \mathrm{OR}$

2. 2-h plasma glucose $\geq 11.1 \mathrm{mmol} / \mathrm{L}$ during Oral Glucose Tolerance Test (OGTT).

The test should be performed as per the WHO,

using a glucose load containing the equivalent of $75 \mathrm{~g}$ anhydrous glucose dissolved in water OR

3. $\mathrm{HbAlc} \geq 48 \mathrm{mmol} / \mathrm{mol}(6.5 \%)$ OR

4. In a patient with classic symptoms of hyperglycaemia or hyperglycaemic crisis,

a random plasma glucose $\geq 11.1 \mathrm{mmol} / 1$

In the absence of unequivocal hyperglycaemia, results should be confirmed with repeat testing [1].

On the other hand, the HbA1c, glycated haemoglobin, is a random blood test, no fasting overnight is required and in Ireland, using DCCT aligned assays, has a very tight coefficient of variability of $<1 \%$. It is not affected by an acute bout of stress, exercise or illness and, because it is a measure of glucose attached to the $\mathrm{N}$-terminal valine residue of each $\beta$ chain of haemoglobin A in the red cell, it gives an indication of glycaemic exposure in the last 2 to 3 months and correlates well with diabetes related complications [3]. The ease of testing and its accuracy has led the HbAlc to become the preferred screening test for diabetes but like any test it has

Maria Tomkins

maria.tomkins@ucdconnect.ie

1 Department of Endocrinology and Diabetes, Beaumont Hospital, Dublin, Dublin, Ireland limitations (Table 2) and the OGTT still has a role to play in screening for diabetes in certain circumstances. Physiological factors such as age and race can elevate the HbA1c. HbA1c concentrations increase by $0.1 \%$ per decade after 30 years of age, thought to be partly due to changes in glycation with age $[4,5]$. HbA1c concentrations are increased in those of African-American, Hispanic and Latino descent compared to their Caucasian counterparts [4]. Diseases which reduce erythrocyte life span, such as haemolytic anaemias or haemoglobinopathies (such as sickle cell trait/disease), result in a reduced $\mathrm{HbAlc}[2,5]$. Chronic kidney disease (CKD) and its impact on $\mathrm{HbAl} \mathrm{c}$ is complicated because of the mixture of iron deficiency anaemia, erythropoietin deficiency and other factors but recent data suggest the $\mathrm{HbAlc}$ underestimates glucose control in patients on dialysis. Iron deficiency and pernicious anaemia result in a falsely elevated $\mathrm{HbA} 1 \mathrm{c}$ due to reduced erythrocyte turnover [4]. Iron deficiency has the additive effect of increasing glycation which further increases the 
Table 1 Factors which influence HbA1c

\begin{tabular}{ll}
\hline Falsely elevated HbAlc & Falsely decreased HbA1c \\
\hline Physiological factors & Iron supplementation \\
Increasing age & Chronic liver disease (due to reduced glycation) \\
Non-Caucasian race & Decreased erythrocyte lifespan \\
Due to decreased red cell turnover & Pregnancy \\
Iron deficiency anaemia & Splenomegaly \\
B12 deficiency anaemia & Haemolytic anaemia \\
Folate deficiency anaemia & Haemoglobinopathies (HbS and HbC) \\
Asplenia & Acute or chronic blood loss \\
Haemoglobinopathies $(\mathrm{HbF}$ and $\mathrm{HbG})$ & Chronic kidney disease \\
Erythropoeitin use & \\
Chronic alcohol intake & \\
Chronic opioid use & \\
\hline
\end{tabular}

HbA1c [6]. The HbA1c will underdiagnose diabetes compared to an OGTT and will miss some patients with early beta cell failure but, because the test is so easy to do, it is likely more patients will be screened at a population level for diabetes than would typically be done with an OGTT.

Following renal transplantation OGTT remains the goldstandard for identifying diabetes mellitus, owing to improved detection, identification of impaired glucose tolerance and lack of reliability of the $\mathrm{HbA} 1 \mathrm{c}$, especially in the early posttransplant period [7]. Alteration of glycation and red cell turnover in cystic fibrosis related diabetes (CFRD) also preclude the use of HbAlc as a screening tool and the OGTT is preferred [8]. However, there is emerging evidence of the use of the HbA1c at a lower threshold of $40 \mathrm{mmol} / \mathrm{mol}$ to diagnose glucose intolerance in this group, as an attempt to reduce the burden of the OGTT in these patients [9]. Use of the HbA1c may also miss the diagnosis in patients with acute hyperglycaemia where the duration of high blood glucose levels is too short to as yet affect the HbAlc. Pregnancy is a state of increased red cell turnover in the second and third trimester and therefore $\mathrm{HbAl} \mathrm{c}$ is not a suitable test to diagnose diabetes in pregnancy [1]. Specifically gestational diabetes is a relatively acute condition and a HbAlc may not accurately reflect hyperglycaemia given it reflects the previous 3 months of glycaemia, therefore currently the recommendation to diagnose gestational diabetes is the OGTT [10].

Clinicians need to be aware of the limitations and concerns with the individual diagnostic tests available to diagnose diabetes. The application of continuous glucose monitoring

Table 2 Conditions which favour FPG/OGTT testing

Conditions which favour diabetes screening with FPG/OGTT

Chronic kidney disease stages $4 / 5$

New-onset diabetes after transplant

Gestational Diabetes Mellitus or diabetes in pregnancy

Cystic-fibrosis related diabetes

Acute onset of diabetes

Diseases and conditions which alter the $\mathrm{HbA1c}$ systems (CGMS) to diagnose diabetes seems to be a next logical step but, expense may inhibit its widespread use. CGMS provides information on fasting, postprandial glucose levels as well as glucose excursions and variability. This provides the diabetologist with information on glucose levels at multiple time-points and facilitates early diagnosis of impaired glucose tolerance or dysglycaemia [11]. Early reports of CGMS as a diagnostic tool show promising results, with superiority to OGTT and HbA1c [11] and particularly in gestational diabetes CGMS would give the clinician and patient a much better picture of foetal glucose exposure than an OGTT.

The HbA1c is increasingly been used to diagnose diabetes and the simplicity of a random blood test will hopefully translate into more patients been screened for diabetes but like every test there are limitations and so the OGTT still has a role to play in certain circumstances to diagnose diabetes.

\section{References}

1. American Diabetes Association. 2 (2018) Classification and diagnosis of diabetes: standards of medical care in diabetes. Diabetes Care 41(Supplement 1):S13-S27. https://doi.org/10.2337/dc18S002

2. Definition and diagnosis of diabetes mellitus and intermediate hyperglycaemia; World Health Organization/International Diabetes Federation 2006. Accessed from: https://www.who.int/ diabetes/publications/Definition\%20and\%20diagnosis $\% 20$ of\% 20diabetes_new.pdf. [Accessed on 10/10/2019]

3. Sacks D (2011) A1c versus glucose testing: a comparison. Diab Care 34(2):518-523

4. Welsh K, Kirkman M, Sacks D (2016) Role of glycated proteins in the diagnosis and management of diabetes: research gaps and future directions. Diab Care 39:1299-1306

5. Campbell L, Pepper T, Shipman K (2019) HbA1c: a review of nonglycaemic variables. Journal of Clinical Pathology 72:12-19

6. Sodi R, McKay K, Dampetla S, Pappachan JM (2018) Monitoring glycaemic control in patients with diabetes mellitus. BMJ 363: k4723

7. Sharif A, Hecking M, de Vries APJ, Porrini E, Hornum M, RasoulRockenschaub S, Krebs G, Berlakovich M, Kautzky-Willer A, Schernthaner G, Marchetti P, Pacini G, Ojo A, Takahara S, Larsen JL, Buddle K, Eller K, Pascual J, Jardine A, Bakker SJL, 
Valderhaug TG, Jenssen TG, Cohney S, Saemann MD (2014) Proceedings from an international consensus meeting on posttransplantation diabetes mellitus: recommendations and future directions. Am J Transplant 14(9):1992-2000

8. Godbout A, Hammana I, Potvin S, Mainville D, Rakel A, Berthiaume Y, Chiasson J-L, Coderre L, Rabasa-Lhoret R (2008) No relationship between mean plasma glucose and glycated haemoglobin in patients with cystic fibrosis-related diabetes. Diabetes Metab 34(6):568-573

9. Burgess J, Bridges N, Banya W, Gyi KM, Hodson M, Bilton D, Simmonds NJ (2016) HbA1c as a screening tool for cystic fibrosisrelated diabetes. J Cyst Fibros 15(2):251-257
10. Siricharoenthai P, Phupong V (2019) Diagnostic accuracy of $\mathrm{HbA} 1 \mathrm{c}$ in detecting gestational diabetes mellitus. J Matern Fetal Neonatal Med. 11:1-4

11. Soliman A, DeSanctis V, Yassin M, Elalaily R, Eldarsy NE (2014) Continuous glucose monitoring system and new era of early diagnosis of diabetes in high risk groups. Indian J Endocrinol Metab. 18(3):274-282

Publisher's note Springer Nature remains neutral with regard to jurisdictional claims in published maps and institutional affiliations. 